\title{
A prática do planejamento em saúde na atenção secundária: uma análise a partir do PMAQ-CEO - $1^{\circ}$ Ciclo
}

\author{
The practice of health planning in secondary care: an analysis from the PMAQ-CEO - 1st Cycle \\ La práctica de la planificación de la salud en la atención secundaria: un análisis basado en el \\ PMAQ-CEO - 1er ciclo
}

Recebido: 24/08/2021 | Revisado: 29/08/2021 | Aceito: 02/09/2021 | Publicado: 05/09/2021

\author{
Janete Maria Rebelo Vieira \\ ORCID: https://orcid.org/0000-0002-7125-9082 \\ Universidade Federal do Amazonas, Brasil \\ E-mail: rebelovieirajm@gmail.com \\ Larissa Neves Quadros \\ ORCID: https://orcid.org/0000-0003-2828-5450 \\ Universidade Federal do Amazonas, Brasil \\ E-mail: lissaquadros_@hotmail.com \\ Isadora Leonília dos Santos Lira \\ ORCID: https://orcid.org/0000-0002-7717-6659 \\ Universidade Federal do Amazonas, Brasil \\ E-mail: isadoraleolira1996@gmail.com \\ Andressa Coelho Gomes \\ ORCID: https://orcid.org/0000-0001-9306-8474 \\ Universidade Federal do Amazonas, Brasil \\ E-mail: andressa.coelho.gomes@gmail.com
}

\begin{abstract}
Resumo
O objetivo do estudo foi avaliar a prática do planejamento nos Centros de Especialidades Odontológicas (CEO) do estado do Amazonas que fizeram adesão ao Programa Nacional de Melhoria do Acesso e da Qualidade dos Centros de Especialidades Odontológicas (PMAQ/CEO) do $1^{\circ}$ Ciclo. Realizou-se um estudo do tipo descritivo e seccional, utilizando-se microdados da avaliação externa do programa, a respeito das avaliações do módulo VIII referente à entrevista com profissionais, direcionado aos gerentes e cirurgiões-dentistas (CD). Os dados foram obtidos e tabulados pelo programa TabWin sendo exportados para o programa Excel. Dos 11 CEO estudados, somente 36,4\% possuíam gerentes que atuavam exclusivamente na função e apenas 1 CEO realizou alguma ação de planejamento nos últimos 12 meses. Apesar de grande parte deles relatarem a realização de reuniões das equipes e procederem à autoavaliação, apenas 4 realizaram o monitoramento e análise das metas por especialidade e avaliaram as ações planejadas/programadas. Concluiu-se que a prática do planejamento nos serviços públicos de atenção secundária em saúde bucal no Amazonas ainda é frágil e incipiente para alcançar a melhoria do acesso e da qualidade dos serviços prestados à população.
\end{abstract}

Palavras-chave: Planejamento em saúde; Assistência odontológica; Atenção secundária à saúde.

\begin{abstract}
The aim of the study was to evaluate the practice of planning at the Centers of Dental Specialties (CEO) in the state of Amazonas that joined the National Program for Access and Quality Improvement of the Centers of Dental Specialties (PMAQ/CEO) of the 1st Cycle. A descriptive and sectional study was performed, using microdata from the external evaluation of the program, in respect of the evaluations of module VIII regarding the interview with professionals, directed to managers and dentists (CD). Data were obtained and tabulated by the TabWin program and exported to the Excel program. Of the 11 CEOs studied, only $36.4 \%$ had managers who worked exclusively in the role and only 1 CEO performed any planning action in the last 12 months. Although a large part of them report the holding of team meetings and proceed with self-assessment, only 4 accomplish the monitoring and analysis of the goals by specialty and evaluated the planned/programmed actions. Thus, it was concluded that the practice of planning in public services of secondary care in oral health in Amazonas is still fragile and incipient to achieve improved access and quality of services provided to the population.
\end{abstract}

Keywords: Health planning; Dental care; Secondary care.

\section{Resumen}

El objetivo del estudio fue evaluar la práctica de la planificación en los Centros de Especialidades Dentales (CEO) del estado de Amazonas que se sumaron al Programa Nacional de Mejoramiento de Acceso y Calidad de los Centros de Especialidades Dentales de 1er Ciclo (PMAQ / CEO). Se realizó un estudio descriptivo y seccional, utilizando 
microdatos de la evaluación externa del programa, sobre las evaluaciones del módulo VIII en cuanto a la entrevista con profesionales, dirigido a gerentes y odontólogos (CD). Los datos fueron obtenidos y tabulados por el programa TabWin y exportados al programa Excel. De los 11 CEO estudiados, solo el 36,4\% tenía gerentes que trabajaban exclusivamente en la función y solo 1 CEO realizó alguna acción de planificación en los últimos 12 meses. Si bien una gran parte de ellos reportó la realización de reuniones de equipo y autoevaluación, solo 4 realizaron el seguimiento y análisis de las metas por especialidad y evaluaron las acciones planificadas / programadas. Se concluyó que la práctica de la planificación en los servicios públicos de atención secundaria de salud bucal en Amazonas es aún frágil e incipiente en cuanto a mejorar el acceso y la calidad de los servicios brindados a la población.

Palabras clave: Planificación de la salud; Cuidado dental; Atención sanitaria.

\section{Introdução}

Planejar consiste em desenhar, executar e acompanhar um conjunto de propostas de ação com vistas à intervenção sobre um determinado recorte da realidade. Por planejamento entende-se um processo de racionalização das ações humanas que consiste em definir proposições e construir a sua viabilidade, com vistas à solução de problemas e atendimento de necessidades individuais e coletivas (Teixeira, 2010).

Por outro lado, a ausência do planejamento faz com que as atividades sejam executadas por inércia, fazendo com que os serviços de saúde funcionem de forma desarticulada, sendo apenas orientados pela noção de qual seja seu papel, reflexo da visão de mundo de cada dirigente ou colaborador, ou de seu entendimento sobre as diretrizes estabelecidas pela política setorial do Estado. Consequentemente, o funcionamento do sistema de saúde torna-se subjetivo, fragmentado e desordenado na medida em que tantas visões e modos de operação existam. Assim, sem o entendimento do objetivo comum de onde se pretende chegar, cada profissional conduzirá e realizará suas atividades a sua maneira, fazendo com que os avanços de uns se neutralizam pelos retrocessos de outros, impossibilitando o ganho de qualidade e a utilização dos recursos de forma mais eficiente (Vieira, 2009).

No processo da implementação do Sistema Único de Saúde (SUS), um importante pilar para a sua consolidação é representado pela descentralização da gestão, concretizada pela transferência de serviços, poder, recursos e responsabilidades aos municípios (Lacerda et al., 2012). Nesse contexto, o planejamento passou a ser considerado como uma importante ferramenta para o efetivo funcionamento do SUS, compondo parte do seu arcabouço legal (Lacerda et al., 2012; Ferreira et al., 2018). O planejamento então é um instrumento de gestão das três esferas de governo, permitindo a definição de objetivos e a organização das ações a serem desenvolvidas, facilitando o acompanhamento, a fiscalização, o controle dos gastos e a avaliação dos resultados obtidos (Lacerda et al., 2012).

Deste modo, para que as ações de planejamento contribuam para o aperfeiçoamento da gestão do SUS, os entes federados devem comprometer-se a realizar processos periódicos de monitoramento e avaliação, orientados pelas diretrizes, objetivos, metas e indicadores assumidos em cada esfera da gestão (Figueiredo et al., 2020). À vista disso, a institucionalização da avaliação em saúde, vem ganhando força em diferentes áreas do setor público em saúde, e assim, iniciativas como o Programa Nacional de Melhoria do Acesso e da Qualidade dos Centros de Especialidades Odontológicas (PMAQ-CEO) e estudos de avaliação de CEO devem ser destacadas, na medida que a avaliação dos serviços oferecidos à população, como uma prática rotineira e continuada nos diversos setores da saúde, contribuirá para que os padrões adequados de qualidade sejam equivalentes nos diferentes contextos do país (Leal; Emi; Araújo, 2021).

Os CEO representam uma das estratégias para ampliar o acesso e qualificar a oferta de serviços na atenção de média complexidade em saúde bucal (Freitas et al., 2016). São caracterizados por serem unidades de referência para as equipes de saúde bucal da Atenção Básica (AB), levando em consideração a realidade epidemiológica de cada região e município e abrangendo objetivos como o combate à perda de tempo, a longa espera por procedimentos e a falta de resolução dos problemas enfrentados pelos usuários do SUS, que necessitam de tratamento odontológico integral (Herkrath et al., 2012; Ministério da Saúde, 2004a). Entretanto, em algumas regiões do país, a implantação desses centros ocorre de maneira ainda 
lenta frente à demanda da população (Herkrath et al., 2012). O estado do Amazonas, que possui 62 municípios, oferta poucos serviços públicos especializados em saúde bucal, apesar das necessidades explicitadas no levantamento epidemiológico de âmbito nacional em saúde bucal para a região (Ministério da Saúde, 2004b; 2012).

Nesse contexto, a avaliação da prática do planejamento nos serviços de saúde é imprescindível, levando-se em conta que este objetiva especificamente melhorar o estado de saúde de uma determinada população, garantindo o acesso com equidade e justiça, bem como agilizar a resposta do sistema de saúde às necessidades percebidas pela comunidade (WHO, 2004). No âmbito da saúde bucal da mesma forma, as ações de planejamento configuram uma ferramenta para encontrar soluções e enfrentar barreiras, como o acesso aos serviços odontológicos, onde se torna essencial o conhecimento quantitativo e qualitativo de dados indicativos à atenção secundária em saúde bucal (Herkrath et al., 2012; Machado et al. 2015). O objetivo do presente estudo foi, portanto, avaliar a prática do planejamento nos Centros de Especialidades Odontológicos, participantes do PMAQ no $1^{\circ}$ ciclo, do estado do Amazonas.

\section{Metodologia}

Um estudo descritivo e seccional com base em dados secundários foi realizado, utilizando-se os microdados da avaliação externa do PMAQ-CEO no $1^{\circ}$ ciclo, relativos a todos os Centros de Especialidades Odontológicas do estado do Amazonas que estabeleceram adesão ao programa. As variáveis do estudo foram selecionadas dentro do módulo de avaliação VIII, denominado "Entrevista com profissionais", direcionado aos gerentes e pelo menos um cirurgião-dentista dos CEO, a fim de obter informações sobre o processo de trabalho da equipe.

Ao total, foram selecionadas 15 variáveis, distribuídas em informações do gerente do CEO: se possuía gerente, quanto tempo atuava, se possuía ou estava em formação complementar; quanto ao planejamento: se realizou alguma atividade de planejamento nos últimos 12 meses, se incluiu CDs, se considerou os cinco itens (as informações do Sistema de Informações Ambulatoriais (SIA), as informações locais de levantamento epidemiológico, as metas de cada especialidade estabelecidas pelo Ministério da Saúde (MS), os desafios apontados a partir da autoavaliação, o envolvimento de organizações da comunidade), se as equipes avaliavam as ações, se realizavam reuniões em equipe e a periodicidade, se o CEO recebeu apoio para o planejamento e organização do processo de trabalho, se o CEO utilizou os quatro recursos principais para a realização do planejamento, se recebeu apoio da gestão na organização do processo de trabalho e por fim, quanto ao processo de autovaliação nos últimos 6 meses.

Os dados foram submetidos a uma análise estatística descritiva. Foram apresentadas as distribuições de frequências das variáveis, apresentando-se os resultados por meio de tabelas. As análises estatísticas foram realizadas pelos programas Tab para Windows (TabWin) e Microsoft Office Excel.

\section{Resultados}

Os dados dispostos no banco do $1^{\circ}$ ciclo do PMAQ CEO em 2013 englobaram a presença de 11 Centros de Especialidades Odontológicas, sendo três na capital Manaus, e oito no interior do estado, nos municípios de Boca do Acre, Iranduba, Itacoatiara, Lábrea, Maués, Parintins, Tefé e São Gabriel da Cachoeira.

Na Tabela 1 são apresentadas as variáveis referentes às informações dos gerentes dos CEO. Observou-se que 54,5\% dos CEO possuíam gerentes com atividade clínica concomitante ao cargo, em contrapartida, apenas uma pequena porcentagem representou aqueles que atuavam exclusivamente como gerente. Quanto ao tempo de atuação frente ao cargo, houve o predomínio de permanência no cargo há menos de um ano. 
Tabela 1. Informações sobre os gerentes dos CEO do estado do Amazonas (2014).

\begin{tabular}{|c|c|c|c|c|c|c|c|c|}
\hline \multicolumn{3}{|c|}{$\begin{array}{l}\text { O CEO possui gerente } \\
\text { N }(\%)\end{array}$} & \multicolumn{4}{|c|}{$\begin{array}{l}\text { Quanto tempo atua como gerente } \\
\qquad N(\%)\end{array}$} & \multicolumn{2}{|c|}{$\begin{array}{l}\text { Possui ou está em formação } \\
\text { complementar } \mathbf{N}(\%)\end{array}$} \\
\hline $\begin{array}{l}\text { Sim, com } \\
\text { atividade clínica }\end{array}$ & $\begin{array}{l}\text { Sim, exclusivo } \\
\text { na função }\end{array}$ & Não & $\begin{array}{l}<1 \\
\text { ano }\end{array}$ & $\begin{array}{c}1-2 \\
\text { anos }\end{array}$ & $\begin{array}{c}3-4 \\
\text { anos }\end{array}$ & 5-9 anos & $\begin{array}{l}\text { Saúde coletiva } \\
\text { Saúde Pública }\end{array}$ & Outros \\
\hline $6(54,5)$ & $4(36,4)$ & $\begin{array}{c}1 \\
(9,1)\end{array}$ & $\begin{array}{c}4 \\
(40,0)\end{array}$ & $\begin{array}{c}3 \\
(30,0)\end{array}$ & $\begin{array}{c}2 \\
(20,0)\end{array}$ & $\begin{array}{c}1 \\
(10,0)\end{array}$ & $5(50,0)$ & $5(50,0)$ \\
\hline
\end{tabular}

Fonte: Elaboração própria a partir do banco do PMAQ-CEO, ciclo 1/2013. Disponível em: https://aps.saude.gov.br/ape/pmaq/ciclo1ceo

Dos 11 CEO analisados, apenas 1 realizou planejamento nos últimos 12 meses, e 7 incluíram os CDs nas ações do planejamento, enquanto nenhum dos CEO considerou os cinco itens no desenvolvimento do planejamento. Em relação à avaliação das ações planejadas/programadas menos da metade das equipes a realizavam. A prática de reuniões das equipes foi comprovada por $7 \mathrm{CEO}$, entretanto, a maior parte deles informou que não tinham periodicidade definida para as reuniões de equipe (Tabela 2).

Tabela 2. Planejamento nos CEO do estado Amazonas (2014).

\begin{tabular}{ll}
\hline Prática do planejamento nos CEOs & N (\%) \\
\hline Realizou planejamento nos últimos 12 meses & $1(9,1)$ \\
O planejamento das ações incluiu os CD & $7(63,4)$ \\
CEO que consideraram os cinco itens no planejamento & $0(0,0)$ \\
Equipes que avaliaram as ações planejadas/programadas & $4(36,4)$ \\
CEO que realizam reunião de equipe & $7(63,4)$ \\
Semanal & $1(9,1)$ \\
Quinzenal & $0(0,0)$ \\
Mensal & $2(18,2)$ \\
Sem periodicidade definida & $4(36,4)$ \\
\hline
\end{tabular}

Fonte: Elaboração própria a partir do banco do PMAQ-CEO, ciclo 1/2013. Disponível em: https://aps.saude.gov.br/ape/pmaq/ciclo1ceo

No que diz respeito ao apoio para a realização do planejamento em saúde, observou-se uma baixa porcentagem de CEO que o recebiam, por outro lado, no que se refere ao apoio da gestão na organização do processo de trabalho, os resultados foram melhores. Notou-se também a nulidade de CEO que utilizavam os quatro recursos principais para a realização do planejamento (Tabela 3). 
Tabela 3. Organização do processo de trabalho, autoavaliação e monitoramento nos CEO do estado do Amazonas (2014).

\begin{tabular}{ll}
\hline Organização do processo de trabalho & N $(\boldsymbol{\%})$ \\
\hline Apoio para o planejamento e organização do processo de trabalho & $5(45,4)$ \\
Utilização dos quatro recursos principais para a realização do planejamento & $0(0,0)$ \\
Apoio da gestão na organização do processo de trabalho & $7(63,4)$ \\
\hline Processo de Autoavaliação e Monitoramento & $\mathbf{N}(\%)$ \\
\hline Foi realizado algum processo de autoavaliação nos últimos 6 meses & $7(63,4)$ \\
Os resultados da autoavaliação são/foram considerados na organização do processo de & $7(63,4)$ \\
trabalho & $4(36,4)$ \\
Monitora e analisa metas por especialidade & \\
\hline
\end{tabular}

Fonte: Elaboração própria a partir do Banco do PMAQ-CEO, ciclo 1, 2013. Disponível em: https://aps.saude.gov.br/ ape/pmaq/ciclo1ceo

Quanto ao processo de autoavaliação dos CEO, mais da metade destes relatou a realização de algum processo de autoavaliação nos últimos 6 meses, e a mesma quantidade considerou estes resultados da autoavaliação na organização do processo de trabalho. Todavia, o monitoramento e análise das metas por especialidade apresentaram baixa porcentagem (Tabela 3).

\section{Discussão}

A organização dos serviços públicos de odontologia no Brasil constitui um grande desafio para a gestão em saúde. A má distribuição dos serviços odontológicos e a baixa capacidade de oferta criou uma demanda reprimida pelos serviços de saúde bucal (Ministério da Saúde, 2004). No estado do Amazonas, o número de CEO implantados até o fim de 2011 pode ser considerado baixo em comparação à expansão destes serviços em outras regiões do país (Figueiredo e Góes, 2009; OPAS, 2006). Nesse período, apenas treze CEO estavam disponíveis para uma população de aproximadamente 2.800 .000 habitantes, sendo importante também ressaltar as características do estado que dificultam a expansão da rede assistencial de saúde como o isolamento geográfico e a falta de recursos financeiros que se concentram em especial na capital (Herkrath et al., 2012). Tais fatores, tornam a prática do planejamento em saúde indispensável dentro dos serviços públicos do estado, a fim de alcançar resultados eficazes em saúde bucal.

Entretanto, o presente estudo verificou que a prática do planejamento ainda não é uma realidade nos CEO do estado do Amazonas. Embora algumas atividades como a autoavaliação pôde ser verificada com mais frequência, outras etapas essenciais para a execução plena do planejamento foram consideradas incipientes, frágeis e algumas até inexistentes, resultados que possuem o potencial de interferir negativamente na qualidade da assistência à saúde bucal para a população.

Apesar da presença do gerente na maioria dos CEO avaliados, parte significativa destes acumulam função clínica e possuem pouco tempo de atuação. Esse cenário demonstra a fragilidade para o cumprimento das ações de planejamento, uma vez que o pouco tempo de atuação sugere alta rotatividade e inexperiência do gerente. Além disso, as atividades de planejamento possivelmente ficam em segundo plano ao dever de cumprir suas metas clínicas. Nossos achados corroboram, mesmo que de forma inversa, aos resultados do estudo de Lucena et al. (2019), que evidenciou que os CEO cujos gerentes atuam exclusivamente na função, possuem um tempo de atuação maior que 2 anos e que tem uma formação complementar na área de gestão pública ou de saúde coletiva tendem a realizar mais atividades de planejamento.

O baixo percentual de CEO sem periodicidade definida para suas reuniões e a nulidade destes que consideram os 
cinco itens do planejamento e os quatro recursos principais para sua execução também chamam atenção, pois são elementos fundamentais no processo de trabalho e tomada de decisões. Em especial o uso dos itens referente à epidemiologia, como os informativos epidemiológicos locais e os dados dos sistemas de informação, haja visto que o sucesso do planejamento, ou seja, a efetividade de seus resultados, mantém relação direta com a qualidade das informações disponíveis (Carnut, 2012). A utilização de dados informacionais no planejamento do SUS está prevista desde sua criação, com a Lei Orgânica de Saúde no 8.080/1990, que preconiza o uso de dados epidemiológicos para estabelecer as prioridades e destinação de recursos, sejam financeiros ou humanos. A epidemiologia se articula profundamente aos princípios e diretrizes do SUS, tendo ampla inserção no processo de gestão, contribuindo para a concretização do planejamento e organização do SUS (Ferreira et al., 2018). Dessa forma, a importância do uso da epidemiologia no âmbito dos serviços de atenção à saúde bucal, é necessária no contexto brasileiro, em virtude da descentralização político-administrativa, da insuficiência de recursos disponíveis e da mudança nos padrões de saúde bucal dos brasileiros (Pattussi; Costa; Tomita, 2006).

Embora a maior parte dos cirurgiões-dentistas terem relatado participar das ações de planejamento e receber apoio da gestão local, o apoio recebido pelas equipes dos CEO para a realização do planejamento e organização do processo de trabalho foi considerado insuficiente, o que reforça o caráter incipiente da prática de um planejamento consolidado e transversal, e implica em um baixo desempenho deste serviço, visto que o desempenho dos CEO é influenciado dentre outros fatores, por formas de organização e gerenciamento dos processos de trabalho (Machado et al., 2015).

Os resultados quanto a realização da autoavaliação nos últimos 6 meses, mostraram-se positivos, com mais da metade dos CEO comprovando seu cumprimento e afirmando considerar seus resultados na organização do processo de trabalho. $\mathrm{O}$ que mostra concordância com os pressupostos do PMAQ-CEO, o qual reconhece a autoavaliação como ponto de partida, na medida que os processos orientados para a melhoria da qualidade têm início na identificação e reconhecimento das dimensões positivas e problemáticas do trabalho da gestão e das equipes de atenção à saúde (Ministério da Saúde, 2013). Entretanto, estes achados conflitam com a baixa porcentagem de CEO que avaliaram as ações planejadas/programas e realizaram o monitoramento e análise das metas pactuadas. Assim, apesar de o planejamento pautar-se na autoanálise e identificação de fragilidades e potencialidades da realidade local por meio da autoavaliação, sem a avaliação e monitoramento das ações programadas, o alcance das transformações necessárias é dificultado. Desse modo, destaca-se a importância do desenvolvimento de uma cultura organizacional no SUS que tenha a avaliação como atividade intrínseca e inseparável do planejamento e da gestão, uma vez que a ênfase no "como" e "quanto" em detrimento do "o que" e "para quê" ou vice-versa, prejudicaria o compromisso em atender às demandas e necessidades das populações (Furtado et al., 2018).

Apesar das limitações da coleta de dados de bases secundárias, como apresentar falhas de registro, estes dados podem oferecer informações válidas e representam uma ferramenta importante para o diagnóstico de situações de saúde com vistas a intervenções mais aproximadas do quadro de necessidades da população, em especial à saúde bucal.

Assim, mesmo com os avanços da prestação dos serviços de saúde bucal como uma função do Estado, identifica-se o uso do planejamento de forma residual, demonstrando uma prática pouco difundida pelos profissionais (Carnut, 2012). Os resultados aqui salientados contribuem para entender o desempenho insatisfatório apresentado pela atenção secundária em saúde bucal no estado do Amazonas, quanto ao cumprimento das metas de produção ambulatorial, apontado em estudo prévio (Herkrath et al., 2012). Sendo assim, este estudo reforça a importância da prática do planejamento em saúde nos CEO como estratégia para melhoria do processo de trabalho e consequente aprimoramento dos serviços especializados em saúde bucal locais.

\section{Conclusão}

Ainda que itens relevantes da prática do planejamento tenham se mostrado positivos, como a realização da 
autoavaliação, a implantação de ferramentas de planejamento nos CEO do estado do Amazonas mostrou-se frágil e incipiente para alcançar a melhoria do acesso e da qualidade dos serviços prestados à população. A prática do planejamento é fundamental na gestão e no cotidiano dos serviços odontológicos em qualquer cenário, e no contexto amazônico, com suas particularidades geográficas e o perfil epidemiológico de doenças bucais, pode ser entendido como imperativo.

\section{Referências}

Carnut. L. (2012). Planejamento e programação de ações em saúde: conceitos, importância e suas influências na organização dos serviços de saúde bucal. $J$ Manag Prim Healh Care 3 (1), 53-61. https://doi.org/10.14295/jmphc.v3i1.118

de Lucena, E. H. G., de Lucena, C. D. R. X., de Goes P. S. A. \& de Sousa M. F. (2019). Condições associadas a atividade de planejamento nos Centros de Especialidade Odontológicas. Univ Odontol. 38 (80), 1-27. https://doi.org/10.11144/Javeriana.uo38-80.caap

Ferreira, J., Celuppi, I. C., Baseggio, L., Geremia, D. S., Madureira, V. S. F \& Souza, J. B. (2018). Planejamento regional dos serviços de saúde: o que dizem os gestores? Saúde e Sociedade 27 (1), 69-79. https://doi.org/10.1590/S0104-12902018170296

Figueiredo, I. D. T., Torres, G. M. C., Cândido, J. A. B., Morais, A. P. P., Pinto, A. G. A. \& Almeida, M. I. (2020). Planejamento estratégico como ferramenta de gestão local na atenção primária à saúde. REFACS 8 (1), 27-38.

Figueiredo, N. \& Góes, P. S. A. (2009). Construção da atenção secundária em saúde bucal: um estudo sobre os Centros de Especialidades Odontológicas em Pernambuco, Brasil. Cad Saúde Pública 25 (2), 259-67.

Freitas, C. H. S. M., Lemos, G. A., Pessoa, T. R. R. F., Araújo, M. F. \& Forte, F. D. S. (2016). Atenção em saúde bucal: avaliação dos centros de especialidades odontológicas da Paraíba. Saúde debate 40 (108), 131-143. https://doi.org/10.1590/0103-1104-20161080011

Furtado, J. P., Campos, G. W. D. S., Oda, W. Y., \& Onocko-Campos, R. (2018). Planejamento e Avaliação em Saúde: entre antagonismo e colaboração. Cadernos de Saúde Pública, 34 (7), e00087917, 1-12. https://doi.org/10.1590/0102-311X00087917

Herkrath, F. J., Herkrath, A. P. C. Q., Costa, L. N. B. S. \& Gonçalves, M. J. F (2012). Desempenho dos Centros de Especialidades Odontológicas frente ao quadro sociodemográfico dos municípios do Amazonas, Brasil, 2009. Saúde debate 37 (96), 148-58.

Lacerda, J. T., Calvo, M. C. M., Berretta, I. Q. \& Ortiga, A. M. B. (2012). Avaliação da gestão para o planejamento em saúde em municípios catarinenses. Ciênc Saúde Coletiva 17 (4), 851-859.

Leal, R. V. S., Emmi, D. T., \& Araújo, M. V. D. A. (2021). Acesso e qualidade da atenção secundária e da assistência em estomatologia no Brasil. Physis: Revista de Saúde Coletiva, 31(2), e310205, 1-23. https://doi.org/10.1590/S0103-73312021310205

Machado, F. C. A., Silva, J. V. \& Ferreira, M. A. F. (2015). Fatores relacionados ao desempenho de Centros de Especialidades Odontológicas. Ciênc Saúde Coletiva 20 (4), 1149-1163. https://doi.org/10.1590/1413-81232015204.00532014

Ministério da Saúde (2004a). Diretrizes da Política Nacional de Saúde Bucal. Brasília. http://bvsms.saude.gov.br/bvs/ publicacoes/politica_nac ional_brasil_sorridente.pdf.

Ministério da Saúde (2004b). Projeto SB Brasil 2003: condições de saúde bucal da população brasileira 2002-2003: resultados principais. https://bvsms.saude.gov.br/bvs/publicacoes/condicoes_saude_bucal.pdf

Ministério da Saúde (2012). SB Brasil 2010: Pesquisa Nacional de Saúde Bucal: resultados principais. https://bvsms.saude.gov.br/bvs/publicac oes/pesquisa_nacional_saude_bucal.pdf

Ministério da Saúde (2013). Autoavaliação para a Melhoria do Acesso e da Qualidade dos Centros de Especialidades Odontológicas: AMAQ-CEO. http://189.28.128.100/dab/docs/portaldab/publicacoes/ AMAQ_CEO.pdf.

Organização Pan-Americana da Saúde (2006). Ministério da Saúde. A Política Nacional de Saúde Bucal do Brasil: registro de uma conquista histórica. Série Técnica Desenvolvimento de Sistemas e Serviços de Saúde, 11. http://dab.saude.gov.br/docs/publicacoes/geral/serie_tecnica_11_port.pdf.

Pattussi, M. P., Costa, J. S. D., \& Tomita, N. E. (2006). O uso da epidemiologia nos serviços de atenção à saúde bucal. In Antunes, J. L. F., Peres, M. A. Epidemiologia da Saúde Bucal (pp. 322-334). Ed. Guanabara Koogan.

Teixeira, C. F. (2010). Planejamento em saúde: conceitos, métodos e experiências. EDUFBA, 161 p.

Vieira, F. S. (2009). Avanços e desafios do planejamento no Sistema Único de Saúde. Ciênc Saúde Coletiva 14 (1), 1565-77. https://doi.org/10.1590/S141381232009000800030

World Health Organization (2004). Regional Office for Africa. Planning and implementation of district health services. 